\title{
A Comparative Analysis on Performance of Mobile IP with Paging Support
}

\author{
Hung Tuan Do and Yoshikuni Onozato \\ Department of Computer Science, Gunma University, Kiryu, Gunma 376-8515, Japan \\ \{hung, onozato\}@nztl.cs.gunma-u.ac.jp
}

\begin{abstract}
This paper presents a comparative analysis on the signaling cost functions of Mobile IP (MIP) with different paging protocols and schemes and investigates constructing optimal paging areas using discrete system model as a mobility model. In wireless mobile Internet, mobile users often visit foreign networks that might be far away from their home networks and the occurrences of their inter-domain movement are relatively rare. In this scenario, our analytical results show that paging, particularly individual paging, can significantly improve the total signaling cost of MIP. We show that Domain paging can bring about considerable cost saving compared to FA (Foreign Agent) paging. Our results also demonstrate the significant advantages of Individual paging over Aggregate paging. The results show that specifying the optimal PA size is critical in saving signaling cost of MIP with paging support.
\end{abstract}

\section{Introduction}

At present, MIP is the current standard protocol for the mobility management at IP layer. Unfortunately, Mobile IP (MIP) was first designed without consideration of the performance efficiency and QoS warranty. We believe that, in addition to the popular approach of localization of location updates, paging is an important approach to improve the performance of MIP [1], [2], [3].

In wireless mobile networks, paging is a process to determine the exact location of a specific Mobile Terminal (MT) in PCSs or a Mobile Host (MH) in Mobile IP that is in stand-by state. Paging service is popularly deployed in wireless WAN for two major benefits: to reduce location update cost and to save power consumption of mobile terminals.

With paging support, an idle $\mathrm{MH}$ performs location update less frequently (at each change of paging area) than it does in the base MIP (at each change of subnet). Thus, the application of paging service introduces two main benefits to MIP: reduce location update cost and save power consumption of MHs. Adversely, paging procedure also generates additional signaling overhead that is paging cost itself and the latency in locating an $\mathrm{MH}$ for packet delivery. There exists a tradeoff between paging cost and registration cost, namely with bigger paging area (PA) size, an $\mathrm{MH}$ tends to update its location less frequently, thus the location update cost is reduced, but the cost of paging over the PA certainly increases and vice versa. Therefore, it is desirable to figure out the optimal paging area size that minimizes the total signaling cost. 
In literature, there is a couple of research works addressing the problem of MIP with paging [1], [2], [3]. In Ref. [3], the authors propose P-MIP (Paging Extensions for MIP). In our taxonomy that is presented in section 2, the paging protocol and paging scheme of P-MIP are very similar to FA paging protocol and Aggregate paging scheme, respectively. The performance of P-MIP is investigated using a combination of analysis and simulation. In analysis, the authors present signaling costs of MIP and P-MIP with the assumptions that paging areas and wireless cells are squareshaped and that user mobility model is Fluid Flow - an aggregate mobility model. The paper then compares the signaling costs of MIP and P-MIP under the effects of the numbers of cells in a paging area. The analytical results show that paging could improve the performance of MIP by reducing the signaling cost in well-designed ranges of parameters such as paging area size and active timer.

In [2], the author analyzes the performance of MIP with the proposed paging schemes by simulation using a modified Random Walk as the user mobility model. In this work, paging areas are assumed to be hexagonal and cellular network is assumed as uniform grid of hexagonal cells. Various paging schemes are considered here, namely mcpf (paging with fixed paging area size), $m c p$, $m c p 1$, and $m c p$ _opt (all are individual paging schemes but with different decision algorithms of paging area update). It is shown that paging can bring about cost gain to MIP and that among paging schemes, individual paging schemes have advantages over mcpf , and among individual paging schemes, mcp_opt can outperforms the other schemes. The performance of Hierarchical MIP (HMIP) with paging is also investigated. The combination of $m c p \_o p t$ paging scheme with HMIP is shown to be the best solution among those considered. It should be noted that, the paging protocol in [2] is FA paging protocol and the individual paging schemes are different from ones in our analysis in PA construction and PA update algorithm.

In [1], the authors first proposed complete paging protocols for MIP, namely Home Agent (HA) paging, Foreign Agent (FA) paging, and Domain Paging. These three paging protocols for MIP are evaluated using simulation with three paging algorithms, namely Fixed Paging, Hierarchical Paging, and Last-Location Paging. In our classification, these paging algorithms belong to aggregate paging, i.e. the paper does not address individual paging schemes. In the simulation, no mobility model is assumed in the simulation, but mobility and call traces are employed instead. Regarding to HA updates and paging latency, the paging protocols and paging algorithms are compared. It is demonstrated that Domain paging performs best and HA paging performs worst among three proposed protocols.

Regarding performance evaluation (by analysis or simulation), some shortcomings of them can be seen. First, none of them compares the performance of MIP with different paging protocols and schemes by analysis. Second, the mobility models (e.g. Random Walk [2], Fluid Flow [3]) and assumptions have severe limitations: subnets are assumed to be squared [3], [1] or hexagonal [2]; movement history is not considered. In the Internet, a subnet takes no specific shape and the distance between two end points should be measured by number of hops between them [4]. Thus, any assumption on the geometric shape of a subnet might be far from realistic. Furthermore, the location (state) of a user depends not only on his current location (state), but also on his movement history (past states). The Fluid Flow model [2] is not a good mobility model for pedestrians or mobile users moving with different velocities. In [1], the 
performance of paging protocols is measured by the number of HA location updates and paging latency. We propose that a more complete signaling cost function be employed to evaluate the performance of MIP with paging support.

A comparative study by simulation of Mobile IPv6 and IP paging for dormant mode location update in the scenarios of macro-cellular and hotspot networks is presented in Ref. [6]. From the initial simulation results for the case of fixed paging area size, the authors concluded that Mobile IPv6 dormant mode location management is comparable to IP paging, and hence, IP paging is unnecessary. However, there is difference in terms of configuration assumptions between this paper and our work. In this paper, a subnet consist of a group of cells and an MH needs to update its location at each subnet change, not at each cell change. In our work and Refs. [1], [3], each wireless cell exists in its own subnet, and in the base MIP, an MH is required to update its location per each inter-subnet (inter-cell) movement. In the sense of location update mechanism of dormant MHs, each subnet as a group of cells in this paper acts in the same way as a PA in our work. Furthermore, macro-cell in the simulation is assumed to be square-shaped, but then, the shape of subnets as a group of such macrocells is not always the case in a spatial Internet scenario. As shown later in our analysis, total signaling cost of MIP with paging (or IP paging) is very sensitive to PA size, and MIP with paging is not always better than the base MIP with an arbitrary PA size. Therefore, PA size is a design parameter, and calculating the optimal PA size is critical in order to minimize the signaling cost of MIP with paging support. Unfortunately, the simulation in this paper is conducted only with fixed PA sizes (4 subnets and 9 subnets).

Moreover, an effort to enhance the performance of MIP is Route Optimization Protocol (IETF RO) [7], which is introduced to solve the problem of triangular routing. Route Optimization is already integrated into MIPv6. In Ref. [8], the authors proposed a method using Markovian Decision model to solve optimization problem of the total cost as the sum of link cost and signaling cost in order to improve further the performance of IETF RO.

The contribution of this paper is as follows. We evaluate the performance of MIP with different paging protocols and paging schemes comparatively by analysis. We address two major limitations above by using Discrete System model [4] as the user mobility model with two distinct features: arbitrary subnet topology and consideration of user movement history. Based on the mobility model and the analysis of the operational models of paging protocols and schemes, we derive the total signaling cost functions of MIP with different paging protocols and schemes. We then analyze the cost functions of MIP with different paging protocols and paging schemes under the impact of varying parameters: PA size, incoming call rate and user dwell time. Also, we determine the optimal PA size by using an iterative algorithm and show that specifying the optimal PA is crucial in saving the signaling cost of MIP with paging.

The remaining sections of this paper are organized as follows. In Section 2, we describe and analyze paging protocols and paging schemes. Section 3 formulates the signaling cost functions of MIP with paging protocols and paging schemes. Our numerical results are presented in Section 4. Finally, Section 5 concludes the paper and mentions some directions of our future works. 


\section{Paging Protocols and Paging Schemes}

In this paper, we differentiate between the two terms: paging protocols and paging schemes. Paging protocol, as defined in [1], determines the node that initiates paging process and messages exchanged among nodes. A complete discussion of paging protocol can be found in [1]. The term "paging scheme" in this paper, on the other hand, determines how paging area and its size are specified.

\subsection{Paging Protocols}

In this analysis, we investigate two protocols: Domain paging and Foreign Agent (FA) paging [1]. For Home Agent (HA) paging [1], HA is the very paging initiator. However, HA is also a potential point of failure and bottleneck in MIP. Moreover, for an $\mathrm{MH}$, the HA might be far away from the foreign network in which the $\mathrm{MH}$ is visiting. Therefore, we argue that initiating a paging process from the HA is not a practical approach. One more thing should be noted is that here we consider MIP in the environment of spatial wireless Internet, not MIP in cellular architecture.

\subsubsection{FA Paging Protocol}

The message flows of MIP with FA paging protocol [1] are shown in Fig. 1. The first packet destined for the MH is tunneled from the HA to the last-registered FA (FA1). Subsequently, FA1 buffers the packet and sends paging request to the other FAs in the PA then these FAs page over the air (message 1).

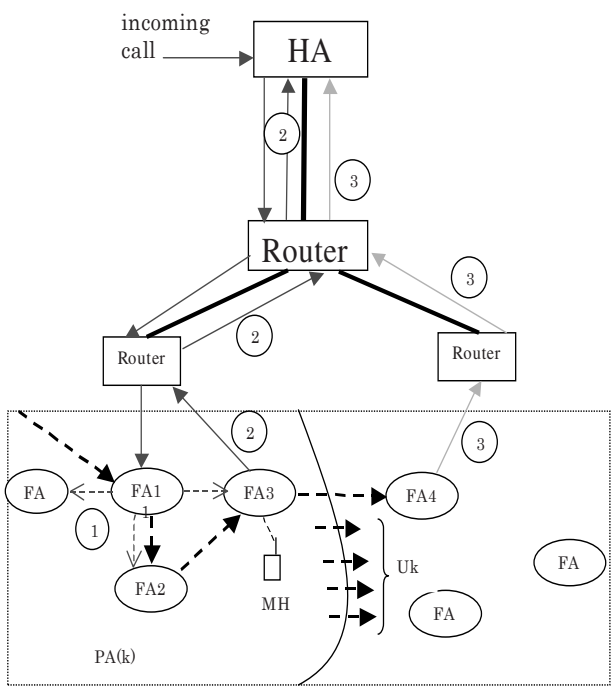

Fig. 1. Message Flows of MIP with FA Paging Protocol

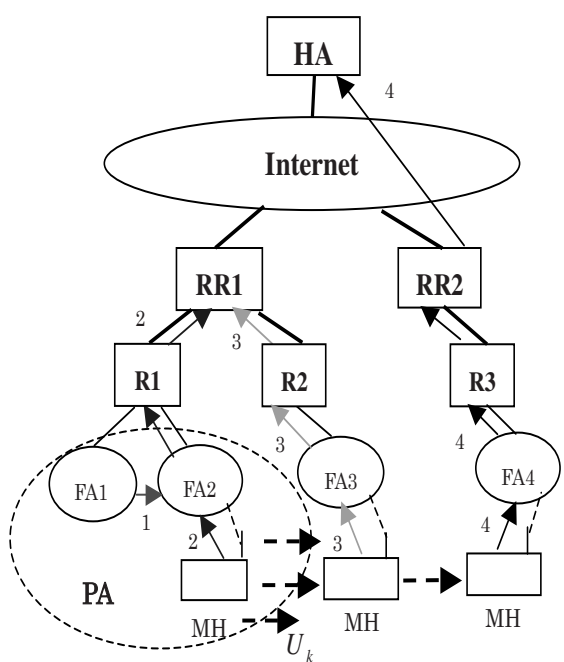

Fig. 2. Message Flows of MIP with Domain Paging Protocol 
The MH registers its new FA with the HA via the location update message following a paging (message 2), which needs to be acknowledged by the HA, and at the same time informs the previous FA. The previous FA forwards the buffered packet to the $\mathrm{MH}$ and subsequently the HA delivers packets to the MH via its new FA. Message 3 indicates the location update binding when the $\mathrm{MH}$ moves out of the paging area with the rate $U_{k}$. Also this message needs to be acknowledged by the HA.

\subsubsection{Domain Paging Protocol}

Domain paging [1] is a router-assisted paging scheme as shown in Figure 2. The domain root router (RR) is the gateway into each domain. When a $\mathrm{MH}$ in standby state moves out of the current paging area with the rate $U_{k}$ but still in the domain, it sends a location update message only to the domain RR (RR1) instead of its HA (message 3), which might be far away (intra-domain mobility). The message is sent hop-by-hop from the MH's FA to the domain root router, thereby creating new routing and paging state on each node (router/BS) in the path.

When an incoming packet destined for the MH in standby mode, a router or FA along the path from the domain root router to the last-registered FA of the MH selects itself to be the page initiator. The page initiator (FA1) then buffers and sends out a page request to all the FAs in the PA and these FAs page over the air (message 1). The $\mathrm{MH}$ replies to the page by sending a page response to the page initiator hop-byhop, thereby updating its location and the route for future packet delivery.

For simplicity, we consider the case where the last-registered FA (FA1) is the page initiator and the page response is always sent to the domain root router to update the routing path to the MH (message 2). Actually, this can be seen as the case of HMIP with FA paging. Only if the MH moves out to another domain (domain 2) then it needs to send a location update binding to its HA (message 4). This is known as interdomain mobility. In fact, the occurrences of inter-domain mobility in the Internet are relatively rare [1] and will be ignored in this paper.

The major difference and also advantage of HMIP with FA paging over MIP with FA paging is the localized location registration of MHs. Moreover, domain paging helps improve significantly the reliability of the system owing to its distributed manner in selecting page initiators.

\subsection{Paging Schemes}

The following paging schemes are considered in this paper:

1. Static Aggregate Paging (SAP): the paging area is designed and fixed by the network administrator for all mobile users.

2. Individual Paging: each user specifies his own paging area (PA) according to his mobility and call pattern, i.e., if the PA size is $\mathrm{k}$ (in terms of the number of subnets covered) then PA will consist of the first $\mathrm{k}$ different visited subnets, similar to the idea of location area construction presented in [4]. In this category, we classify Individual paging further as follows:

3. Static Individual paging (SIP) scheme: The optimal PA size (in terms of the number of subnets covered) $\mathrm{k}_{\mathrm{opt}}$ is pre-computed before communications and will not change during the communications. 
4. Dynamic Individual paging (DIP) scheme: $\mathrm{k}_{\mathrm{opt}}$ is adaptive to the user's current mobility and call parameters.

\section{Total Signaling Cost}

\subsection{Assumption and Parameters}

All parameters and their notations used in our analysis are shown in Table 1.

Table 1. List of parameters

\begin{tabular}{|l|l|l|}
\hline Parameters & \multicolumn{1}{|c|}{ Meanings } & \multicolumn{1}{|c|}{ Units } \\
\hline$k$ & Size of paging area & subnets \\
\hline$\lambda$ & Incoming call/packet rate & calls/minute \\
\hline$U_{k}$ & $\begin{array}{l}\text { Location update rate per MH due to its } \\
\text { movement }\end{array}$ & updates/minute \\
\hline$C_{p}$ & Paging cost in a subnet per call & bytes/paging/subnet \\
\hline$C_{u}$ & Location update cost per hop & bytes/update/hop \\
\hline$d_{m h}$ & MH-HA distance (number of hops) & hops \\
\hline$d_{m g}$ & MH-GW distance (number of hops) & hops \\
\hline$d_{f p}$ & New FA - last-registered FA distance & hops \\
\hline$T_{d}$ & $\begin{array}{l}\text { Dwell time (residence time) of MH in a } \\
\text { subnet }\end{array}$ & minutes \\
\hline$\alpha$ & Wired communication cost weight & \\
\hline$\beta$ & $\begin{array}{l}\text { Cost weight ratio of wireless to wired } \\
\text { communication }\end{array}$ & \\
\hline
\end{tabular}

In this paper, we make the following assumptions:

1. The MH-HA distance $d_{m h}$ and MH-GW distance $d_{m g}$ are assumed as fixed during the time of investigation.

2. Successive calls are not overlapped, i.e. the $\mathrm{MH}$ always accomplishes a call then turns into standby mode before the next call arrives. With this assumption, the $\mathrm{MH}$ is paged at each incoming call.

3. The last-registered FA is the paging initiator in Domain Paging and the MH always send its page response to the domain RR instead of the paging initiator. We also assume that all $\mathrm{N}$ subnets considered in our mobility model are located within a domain; and inter-domain mobility is so rare [1] that it can be ignored in our analysis. 


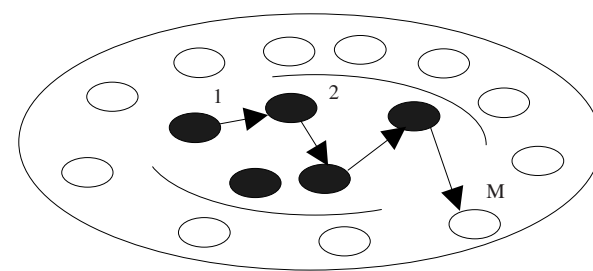

Fig. 3. SAP scheme model

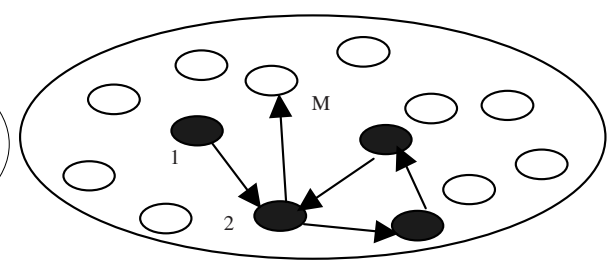

Fig. 4. Individual Paging scheme model

\subsection{PA-Boundary-Crossing Rates}

Our mobility model is adapted from the Discrete System model [4]. We assume that each $\mathrm{MH}$ can move randomly among $\mathrm{N}$ subnets and the PA consists of $k$ subnets. The MH moves from one subnet to any of $(\mathrm{N}-1)$ others with the equal probability $1 /(\mathrm{N}-1)$. The action an MH moves out of a subnet is called "a movement". Let M be a random variable so that the MH moves out of the PA at movement M [4]. This model well captures the spatial nature of the Internet: a subnet can take an arbitrary shape.

In SAP scheme, the PA and its size are pre-defined and the same for all MHs. Similar to [4], the expectation of M in SAP can be derived:

$$
E\left[M_{S A P}\right]=1+\frac{N-1}{N-k} .
$$

Now, the PA-boundary-crossing rate $U_{k}$, which is exactly the HA update rate due to the movement of the $\mathrm{MH}$, can be obtained (where $T_{d}$ is the average dwell time):

$$
U_{k}=\frac{1}{E\left[M_{S A P} T_{d}\right.} .
$$

Contrary to SAP, in Individual Paging, PA and its size are specified adaptively for each MH. PA will comprise the first different $k$ subnets visited by the MH, given PA size $k$. Similar to [4], the expectations of $\mathrm{M}$ in these schemes are:

$$
E\left[M_{S I P}\right]=E\left[M_{D I P}\right]=1+(N-1) \sum_{i=1}^{k} \frac{1}{N-i} .
$$

The PA-boundary-crossing rate $U_{k}$ in this scheme can be obtained:

$$
U_{k}=\frac{1}{E\left[M_{S I P}\right] T_{d}}=\frac{1}{E\left[M_{D I P}\right] T_{d}} .
$$




\subsection{Derivation of Signaling Cost Functions}

\subsubsection{MIP with FA Paging Protocol}

In this analysis, for the sake of comparison, it is not necessary to consider the cost of packet delivery as it is similar to the process in MIP. From our analysis of message flows in MIP with FA paging protocol above, the total signaling cost function comprises of three elements: paging cost $P$ (message 1), the cost of location update following each page $U_{1}$ (message 2) and the cost of location update due to the movement of the $\mathrm{MH}_{2}$ (message 3).

\section{a. Paging Cost}

Paging cost consists of the cost of paging via air interfaces of all $k$ subnets in the paging area and the cost of wired communication when the last-registered FA contacts other FAs in the paging area via wired links and routers. Let $N_{r}$ be the number of routers via that the paging initiating FA needs to send packets to other FAs. The paging cost is thus:

$$
P=\lambda\left[k C_{p}+(k-1) \alpha C_{u}+N_{r} \alpha C_{u}\right] .
$$

For simplicity, we assume that all FAs in current paging area are connected via one router, e.g. when there is only one level of hierarchy of FAs. The paging initiating FA contacts the router then the router will contact $(k-1)$ other FAs in the paging area. Consequently, the paging cost is simplified as follows:

$$
P=k \lambda\left(C_{p}+\alpha C_{u}\right)
$$

\section{b. Cost of Location Update Following Each Page}

The registration of the MH's new FA to the HA with acknowledgment requires the cost of $2 \alpha C_{u}\left(d_{m h}+\beta\right)$. Moreover, the communication cost when the new FA contacts the last FA and the last FA forwards the buffered packet to the new FA is $2 d_{f p} \alpha C_{u}$. Totally, the cost of location update following an incoming call can be obtained as:

$$
U_{1}=2 \lambda \alpha C_{u}\left(d_{m h}+d_{f p}+\beta\right)
$$

With the assumption of one level hierarchy of FAs, we have $d_{f p}=2$. More generally, we can assume $d_{f p}=\sqrt{k}$ [3] where $k$ is the number of subnets in the paging area. 
c. Cost of Location Update Due to the Movement of the MH

Whenever an MH moves out of its current paging area, it is required to register its new location (FA) with its HA. We denote $U_{S A P_{-} 2}$ and $U_{I P_{-} 2}$ as the location update costs of SAP and Individual paging, respectively. From (1), (2), (3) we can obtain:

$$
\begin{aligned}
& U_{S A P_{-} 2}=2 \alpha C_{u}\left(d_{m h}+\beta\right) \frac{1}{E\left[M_{S A P}\right] T_{d}}=2 \alpha C_{u}\left(d_{m h}+\beta\right) \frac{N-k}{(2 N-k-1) T_{d}} . \\
& U_{I P_{-} 2}=2 \alpha C_{u}\left(d_{m h}+\beta\right) \frac{1}{E\left[M_{I P}\right] T_{d}}=2 \alpha C_{u}\left(d_{m h}+\beta\right) \frac{1}{\left[1+(N-1) \sum_{i=1}^{k} \frac{1}{N-i}\right] T_{d}} .
\end{aligned}
$$

\section{d. Total Cost Function}

The total signaling cost functions of MIP with FA paging protocol and SAP, SIP and DIP are denoted $S_{S A P}, S_{S I P}$ and $S_{D I P}$, respectively. For each scheme, the total signaling cost is the summation of three elements described above. Accordingly, we have:

$$
\begin{gathered}
S_{S A P}\left(k, \lambda, T_{d}\right)=P+U_{1}+U_{S A P_{-} 2} . \\
S_{S I P}\left(k, \lambda, T_{d}\right)=S_{D I P}\left(k, \lambda, T_{d}\right)=P+U_{1}+U_{I P_{-} 2} .
\end{gathered}
$$

\subsubsection{MIP with Domain Paging Protocol and Hierarchical MIP with FA Paging Protocol}

As we argued before, MIP with Domain paging in this scenario is similar to HMIP with FA Paging in the faucet of signaling cost, and thus the analytic results here are applicable to both of them.

Similar to MIP with FA paging, the signaling cost of MIP with Domain paging comprises of three elements (Fig 2): paging cost $P$ (message 1), the cost of location update following each page $U_{D_{-} 1}$ (message 2 ) and the cost of location update due to the movement of the $\mathrm{MH} \mathrm{U}_{2}$ (message 3).

\section{a. Paging Cost}

This cost element in MIP with Domain paging is exactly similar to that of MIP with FA paging as in equation (5).

\section{b. Cost of Location Update Following Each Page}

In MIP with Domain paging, following a page, the MH sends its page response message to the domain RR instead of the HA, hence, similar to (6), we have: 


$$
U_{D_{-} 1}=2 \lambda \alpha C_{u}\left(d_{m g}+d_{f p}+\beta\right)
$$

\section{c. Cost of location update due to the movement of the MH}

We denote $U_{S A P_{-} D_{-} 2}$ and $U_{I P_{-} D_{-} 2}$ as the costs of location update due to the movement of the MH in SAP scheme and Individual paging scheme, respectively. When the MH moves out of its current PA, it makes a location binding update to the domain RR, so similar to (7) and (8), $U_{S A P_{-} D_{-} 2}$ and $U_{I P_{-} D_{-} 2}$ are as follows:

$$
\begin{gathered}
U_{S A P_{-} D_{-} 2}=2 \alpha C_{u}\left(d_{m g}+\beta\right) \frac{N-k}{(2 N-k-1) T_{d}} . \\
U_{I P_{-} D_{-} 2}=2 \alpha C_{u}\left(d_{m g}+\beta\right) \frac{1}{\left[1+(N-1) \sum_{i=1}^{k} \frac{1}{N-i}\right] T_{d}} .
\end{gathered}
$$

Let $S_{S I P_{-} D}$ and $S_{D I P_{-} D} S_{S A P_{-} D}$ be the total signaling cost functions of MIP with Domain paging protocol and SIP, DIP, and SAP, respectively. They can be computed as the summation of three cost elements above as follows:

$$
\begin{gathered}
S_{S A P_{-} D}\left(k, \lambda, T_{d}\right)=P+U_{D_{-} 1}+U_{S A P_{-} D_{-} 2} . \\
S_{S I P_{-} D}\left(k, \lambda, T_{d}\right)=S_{D I P_{-} D}\left(k, \lambda, T_{d}\right)=P+U_{D_{-} 1}+U_{I P_{-} D_{-} 2} .
\end{gathered}
$$

\section{Numerical Results}

We now investigate and compare the performance of the paging protocols and schemes under the impact of varying parameters by evaluating the total signaling cost functions. Our performance analysis uses the parameters with numerical values listed in Table 2. This set of values represents a typical situation in a system running MIP with paging support: an $\mathrm{MH}$ is visiting a foreign network far away from its home network; the values of $\alpha$ and $\beta$ are selected to capture the fact that cost weight of wireless link is higher than that of wired link.

For comparison purpose, we should consider all the four combinations paging protocols (two protocols) and paging schemes (two schemes). Fortunately, because paging protocols and paging schemes in this paper are orthogonal, we will actually investigate two combinations. More specifically, first we compare SAP scheme with Individual paging schemes (in FA paging protocol), then we investigate Domain paging protocol and FA paging protocol (both with Individual paging scheme). It 
should be noted that the pure MIP is considered to be a special case of MIP with paging when paging area size $k=1$.

In SAP, the total signaling cost function with respect to $\mathrm{PA}$ size $k$ is a convex function, therefore, any PA size might be far from being optimal. In Individual Paging schemes, however, the total signaling cost functions are concave, and we can obtain the optimal PA size using the iterative algorithm in [5].

Table 2. Numerical values of parameters

\begin{tabular}{|c|c|c|c|c|c|c|c|}
\hline$N$ & $d_{m h}$ & $d_{m g}$ & $d_{f p}$ & $C_{p}$ & $C_{u}$ & $\alpha$ & $\beta$ \\
\hline 30 & 30 & 10 & 2 & 3.0 & 1.0 & 1.0 & 2.0 \\
\hline
\end{tabular}

\subsection{SAP Scheme vs. Individual Paging Scheme}

\subsubsection{Impact of Varying Dwell Time}

Figure 5 shows the total signaling cost of Individual paging vs. that of SAP (both with FA paging) with respect to PA size $\mathrm{k}$. The incoming call rate is fixed $\lambda=0.5$ while the dwell time varies: $T_{d}=0.2 ; 0.5 ; 20.0$. It can be observed that the total signaling cost of Individual paging can be much lower than that of SAP. Particularly, when the mobility of the $\mathrm{MH}$ is high, i.e., Td is small, the benefit of Individual paging over SAP is significant. Up to $58 \%$ of the signaling cost can be saved by using Individual paging instead of SAP. Both paging schemes can have much better performance than that of MIP when $T_{d}$ is relatively small, i.e., high mobility. But when $T_{d}$ is very high such as $T_{d}=20$, i.e., when the MH is almost not mobile, Mobile IP is better. This is because MIP with paging no longer enjoys its advantage of reducing location update cost due to the movement of the $\mathrm{MH}$ (since the $\mathrm{MH}$ is almost not moving) whereas the paging cost to locate it is still the same if the PA size stays unchanged. In this case, SAP scheme and Individual paging scheme are almost the same in terms of signaling cost.

In Fig. 6, the dwelling time is fixed $T_{d}=0.2$ while the incoming call rate varies $\lambda$ $=0.4 ; 3.0$. It can be seen from the graphs that Individual paging schemes induce lower signaling cost than that of SAP scheme. Actually, the different element in the total signaling cost functions of them is the cost of location update due to the movement of the $\mathrm{MH}$ (U2) while the paging cost $\mathrm{P}$ and the cost of location update following a paging U1 are the same. Hence, the difference in the signaling costs of Individual paging and SAP depends on the mobility of the user, which is expressed by Td, but not on the incoming call rate $\lambda$.

Nevertheless, when $\lambda$ is relatively high, e.g. $\lambda=10.0$, the paging cost $\mathrm{P}$ and the location update cost $\mathrm{U} 1$ become the dominating elements in the total signaling cost function, and thus, the difference between Individual paging and SAP in terms of signaling cost gets to be insignificant.

These results are well expected since each MH has its own mobility and call arrival patterns and thus has its own optimal network configuration as we argued before. 
However, the benefit of individual paging comes with the price of high calculation load on the MH itself. In Static aggregate paging, the computation is usually performed by the system and based on the average values of parameters of all mobile users and the result is for all these users. In contrast, the calculation is a burden for MHs in Individual paging and it may reduce the advantage of power saving brought about by paging. The analysis results also show that the total cost function is very sensitive to PA size. Therefore, determining he optimal PA size is crucial in saving total cost of MIP with paging.

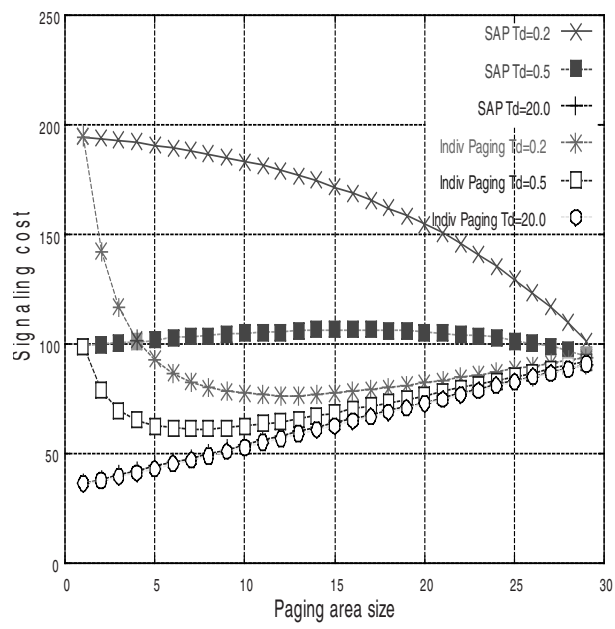

Fig. 5. Total signaling cost vs. PA size for SAP and Individual paging with varying $T_{d}$

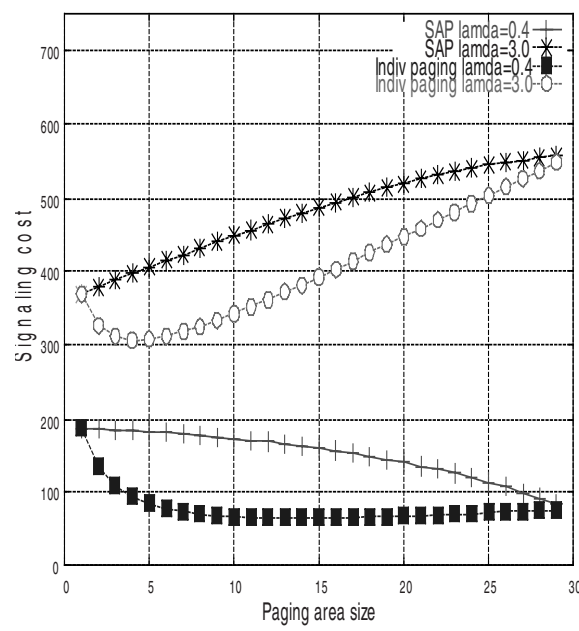

Fig. 6. Total signaling cost vs. PA size for SAP and Individual paging with varying $\lambda$

\subsection{Domain Paging Protocol vs. FA Paging Protocol}

\subsubsection{Impact of Varying Dwell Time}

In Figure 7 , the incoming call rate is fixed $\lambda=0.5$ while user dwell time varies $T_{d}=0.2 ; 0.5$. Domain paging is shown to offer considerably lower signaling cost than that of FA paging, and the difference is even bigger when user mobility is high, i.e. low dwell time.

\subsubsection{Impact of Varying Incoming Call Rate}

The signaling cost of MIP with Domain paging protocol and that of MIP with FA paging protocol (both with Individual paging scheme) are shown against paging area size $\mathrm{k}$ in Fig. 8. $T_{d}$ is fixed and equal to 0.2 while $\lambda$ varies: $\lambda=0.8 ; 3.0$. From the figure, the total signaling load of MIP with Domain paging is significantly lower than that of MIP with FA paging, especially when the incoming call rate is high. 
The advantage actually comes from the benefit of domain-based localization of location registration of mobile users in MIP with Domain paging. Our results demonstrate that Domain paging can offer up to more than $50 \%$ cost saving compared with FA paging. The gain of Domain paging over FA paging depends on both user dwell time $T_{d}$ and incoming call rate $\lambda$. This result is explicable from the formulas of their total signaling cost functions. The different elements of the two protocols are U1 and $\mathrm{U} 2$, which are functions of $\lambda$ and $T_{d}$, respectively.

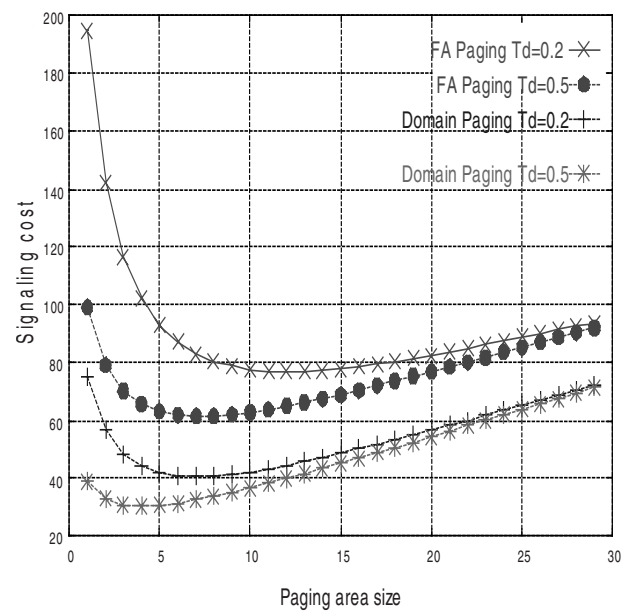

Fig. 7. Total Signaling Cost vs. PA size for Domain paging and FA paging with varying $T_{d}\left(\lambda=0.5 ; T_{d}=0.2 ; 0.5\right)$

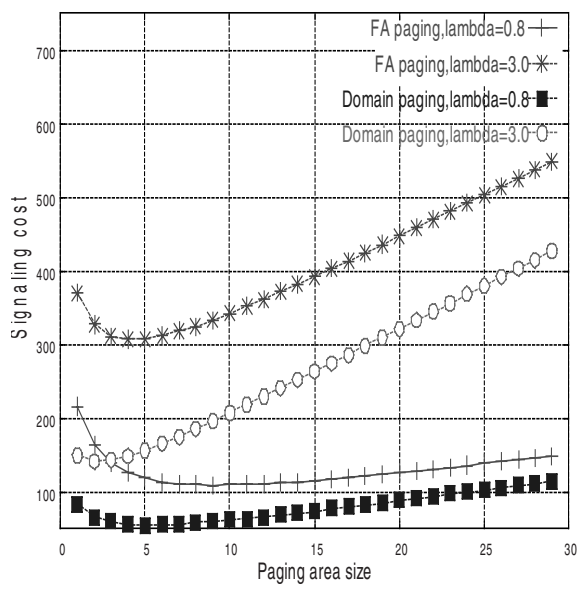

Fig. 8. Total Signaling Cost vs. PA size for Domain paging and FA paging with varying $\lambda\left(T_{d}=0.2 ; \lambda=0.8 ; 3.0\right)$

\section{Conclusions}

In this paper, we have investigated the performance of different paging protocols and schemes with MIP, based on their total signaling cost functions. We have also shown how to construct the optimal PA and how important it is in saving the signaling cost of MIP with paging. Our analysis results show that paging can be an effective approach to improve MIP. Up to $60 \%$ cost saving can be acquired. However, paging service is not a good choice for very low mobility users. Individual paging can significantly outperform SAP and the cost saving may be as high as $58 \%$. The results also demonstrate that Domain paging can enjoy much lower signaling cost than that of FA paging (up to $50 \%$ cost saving). The impact of various user parameters on the total signaling cost is also investigated thoroughly in our analysis.

In our future works, we will consider other mobility models and assumptions. Specifically, we will investigate more general patterns of call arrival and call duration such as Poisson distribution of incoming calls. This also includes the case of over- 
lapped incoming calls. Other mobility models such as Random Walk and Markov models will be taken into account.

\section{References}

1. R. Ramjee, L. Li, T. L. Porta and S. Kasera, "IP Paging Services for Mobile Hosts," Wireless Network 8, 427-441, 2002.

2. C. Castellucia, "Extending Mobile IP with Adaptive Individual Paging: A Performance Analysis," MobilCom, Volume 1, Number 2.

3. X. Zhang, J. Castellanos, and A. Campbell, "Design and Performance of Mobile IP Paging," ACM Mobile Networks and Applications (MONET), Special issue on Modeling Analysis and Simulation of Wireless and Mobile Systems, Vol. 7, No. 2, March 2002.

4. J. Xie, and Ian F. Akyildiz, "A Novel Distributed Dynamic Location Management Scheme for Minimizing Signaling Costs in Mobile IP," IEEE Trans. on MobilCom, Vol. 1, No. 3, Jul-Sept 2002.

5. H.Xie, S. Tabbane, and D. J. Goodman, "Dynamic Location Area Management and Performance Analysis," Proc. 43 ${ }^{\text {rd }}$ IEEE VTC, pp. 536-539, 1993.

6. J. Kempf, and P. Mutaf, "IP Paging Considered Unnecessary: Mobile IPv6 and IP Paging for Dormant Mode Location Update in Macrocellular and Hotspot Networks," IEEE Wireless Communications and Networking Conference, New Orleans, Louisiana, USA, March 2003.

7. C. Perkins and D. Johnson, "Route Optimization in Mobile IP," Internet Draft, Internet Eng. Task Force (IETF), Nov. 2000.

8. Young J. Lee and Ian F. Akyildiz, "A New Scheme for Reducing Link and Signaling Costs in Mobile IP,” IEEE Transactions on Computer, Vol. 52, No. 6, June 2003. 